\title{
ON THE EXISTENCE OF MEROMORPHIC FUNCTIONS WITH PRESCRIBED ZEROS, ONES AND POLES
}

\author{
JÖRG WINKLER
}

1. Introduction. In a lecture [5] given 1966 in honour of Weierstraß, Rolf Nevanlinna treated the following problem: Let three sequences $\left\{a_{\mu}\right\},\left\{b_{\mu}\right\}$, and $\left\{c_{\mu}\right\}$ be given in the complex plane $C$. Does there exist an entire function with exactly the zeros $a_{\mu}$ and the ones $b_{\mu}$ or a meromorphic function with also the poles $c_{\mu}$ ? Nevanlinna mentioned that, to his knowledge, no method for the solution of the described problem was known.

The introduction of Picard sets by Olli Lehto in 1958 is connected with the problem stated by Nevanlinna. A set $E$ contained in $C$ is called a Picard set of entire (respectively meromorphic) functions if any entire (respectively meromorphic) function takes in $C \backslash E$ all values of the extended complex plane $\hat{C}$ with at most two exceptions. Lehto proved in [4] the existence of Picard sets for entire as well as for meromorphic functions. It is obvious that the problem above has no solution if the points $a_{\mu}$ and $b_{\mu}$, or respectively, $a_{\mu}, b_{\mu}$, and $c_{\mu}$, are contained in a Picard set. Sufficient criteria for Picard sets have been given after Lehto by J. M. Anderson, I. N. Baker, J. Clunie, S. Liverpool, K. Matsumoto, S. Toppila and the author (see e.g. [1], [2], [3], [6], [10], [11], [12], [13], [14], [15]).

In [14] the author gave also sequences of points that are not contained in any Picard set and cannot be the union of all the zeros and ones of any entire function.

In 1973 the definition of zero-one-sets was introduced by L. A. Rubel and C. C. Yang [9]: Each pair $\left(\left\{a_{\mu}\right\},\left\{b_{\mu}\right\}\right)$ of sequences of points is called a zero-one-set of entire functions if there exists an entire function $f$ such that $\left\{a_{\mu}\right\}$ are all the zeros and $\left\{b_{\mu}\right\}$ are all the ones of $f$.

The construction of zero-one-sets is thus a solution of the problem stated by Nevanlinna. By the above mentioned result it is obvious that there are pairs of sequences of points that are not zero-one-sets. Results of M. Ozawa [7] and the author [16] explain why the construction of zero-one-sets is so very hard.

In brief, Ozawa proved: If one removes a finite number of points from a given zero-one-set then one cannot expect that the resulting set is a zero-one-set of the entire functions. Briefly, the author proved: If one moves some of the points of a given zero-one-set slightly, then the resulting set of point sequences is not zero-one-set of the entire functions. 
In the proofs of these results by Ozawa and the author essential use is made of the fact that entire functions are concerned. The aim of this paper is to extend the result given by the author about zero-one-sets for entire functions to the case of zeroone-infinity-sets of meromorphic functions. Here we mean by zero-one-infinity-set any triple $\left(\left\{a_{\mu}\right\},\left\{b_{\mu}\right\},\left\{c_{\mu}\right\}\right)$ of sequences of points such that there exists some meromorphic function with zeros $a_{\mu}$, ones $b_{\mu}$, and poles $c_{\mu}$. We will get this result under certain conditions concerning $\left\{a_{\mu}\right\},\left\{b_{\mu}\right\}$ and $\left\{c_{\mu}\right\}$. In the case that these conditions are not fulfilled we show by examples that the result for entire functions is not transferable to meromorphic functions. In this connection we will see that such examples do not appear for entire functions.

2. Further notations and statement of results. Throughout this paper we will use the common notations $T(r, f), m(r, f), N(r, f), \bar{N}(r, f), \ldots$. Especially we will use the notation $N(r, a)=N(r, 1 /(f-a))$ and extend its use as follows: For any sequence $\left\{a_{v}\right\}$ introduce the notation

$$
N\left(r,\left\{a_{v}\right\}\right)=\int_{0}^{r} \frac{n\left(t,\left\{a_{v}\right\}\right)-n\left(0,\left\{a_{v}\right\}\right)}{t} d t+n\left(0,\left\{a_{v}\right\}\right) \log r,
$$

where $n\left(t,\left\{a_{v}\right\}\right)$ counts the number of $a_{v}$ with $\left|a_{v}\right| \leqq t$. (So $n\left(t,\left\{a_{v}\right\}\right)=\max _{\left|a_{v}\right| \leqq t} v$ holds in the case $\left|a_{1}\right|<\left|a_{2}\right|<\left|a_{3}\right|<\ldots$.) With respect to the sequence $\left\{a_{v}\right\}$ we apply the definition of the exponent of convergence as

We can now state

$$
\inf \left\{\left.x\left|\sum_{v=1}^{\infty}\right| a_{v}\right|^{-\varkappa}<\infty\right\}=\limsup _{r \rightarrow \infty} \frac{\log N\left(r,\left\{a_{v}\right\}\right)}{\log r} .
$$

Theorem 1. Let $f$ be a meromorphic function of finite order $\varrho \neq 1,2, \ldots$, with the zeros $a_{1}, a_{2}, \ldots$, the ones $b_{1}, b_{2}, \ldots$, and the poles $c_{1}, c_{2}, \ldots$ where these zeros, ones, and poles are supposed to be simple. We suppose that with some real $d>0$, some real $\tau>1$ and some real $r_{0} \geqq 0$ the relations

$$
0<d<\frac{N(r, 1)}{N(r, 0)+N(r, f)} \text { for all } r \geqq r_{0}
$$

and

$$
\left|x_{\lambda}-x_{\mu}\right| \geqq 2 \exp \left(-\left|x_{\mu}\right|^{e+\frac{1}{4}(\tau-1)}\right) \quad \text { for } \quad x_{\lambda}, x_{\mu} \in \bigcup_{v=1}^{\infty}\left\{a_{v}, b_{v}, c_{v}\right\}
$$

hold. Further let three sequences $\left\{a_{v}^{\prime}\right\},\left\{b_{v}^{\prime}\right\}$ and $\left\{c_{v}^{\prime}\right\}$ be given with

$$
\left\{\begin{array}{l}
\left|a_{v}-a_{v}^{\prime}\right|=O\left(\exp \left(-\left|a_{v}\right|^{2 \varrho+\tau}\right)\right), \\
\left|b_{v}-b_{v}^{\prime}\right|=O\left(\exp \left(-\left|b_{v}\right|^{2 \varrho+\tau}\right)\right), \\
\left|c_{v}-c_{v}^{\prime}\right|=O\left(\exp \left(-\left|c_{v}\right|^{2 \varrho+\tau}\right)\right) .
\end{array}\right.
$$

Then there exists no meromorphic function $g, g \neq f$, with the zeros $a_{1}^{\prime}, a_{2}^{\prime}, \ldots$, the ones $b_{1}^{\prime}, b_{2}^{\prime}, \ldots$, and the poles $c_{1}^{\prime}, c_{2}^{\prime}, \ldots$ (where these zeros, ones, and poles are all simple). 
Let us call a "triple of point sequences of order $\varrho$ " each triple $\left(\left\{a_{v}\right\},\left\{b_{v}\right\},\left\{c_{v}\right\}\right)$ of three point sequences with the property that the maximum of the exponents of convergence of these three sequences is equal to $\varrho$. For the linear space of all triples of point sequences of order $\varrho$ with $\varrho \neq 1,2,3, \ldots$ Theorem 1 gives: The zero-oneinfinity sets of meromorphic functions of order $\varrho$ have no accumulation point in this space where the neighbourhoods of the triple $\left(\left\{a_{v}\right\},\left\{b_{v}\right\},\left\{c_{v}\right\}\right)$ are given by all triples $\left(\left\{a_{v}^{\prime}\right\},\left\{b_{v}^{\prime}\right\},\left\{c_{v}^{\prime}\right\}\right)$ such that $\left|a_{v}-a_{v}^{\prime}\right| \leqq r_{v}, \quad\left|b_{v}-b_{v}^{\prime}\right| \leqq r_{v},\left|c_{v}-c_{v}^{\prime}\right| \leqq r_{v}$ and $\sum_{v=1}^{\infty} r_{v}<\infty$.

From Theorem 1 the question arises to what extent the conditions (2.1) and (2.2) are necessary. In the case of supposition (2.1) it is obvious that here the zeros, ones, and poles can be arbitrarily permutated. Theorem 2 shows that the supposition (2.2) is essentially sharp.

Theorem 2. For any real $\varrho \geqq 0$ there exists a meromorphic function $f$ of order $\varrho$ with zeros $a_{1}, a_{2}, \ldots$, ones $b_{1}, b_{2}, \ldots$, and poles $c_{1}, c_{2}, \ldots$ and with the following properties:

(1) All zeros, ones, and poles are simple.

(2) For all $v \neq \mu$

hold.

$$
\begin{aligned}
& \left|a_{v}-a_{\mu}\right| \geqq \exp \left(-\left|a_{\mu}\right|^{e+\frac{1}{4}(\tau-1)}\right), \\
& \left|b_{v}-b_{\mu}\right| \geqq \exp \left(-\left|b_{\mu}\right|^{e+\frac{1}{4}(\tau-1)}\right), \\
& \left|c_{v}-c_{\mu}\right| \geqq \exp \left(-\left|c_{\mu}\right|^{e+\frac{1}{4}(\tau-1)}\right)
\end{aligned}
$$

(3) With some $d>0, \tau>1, r_{0} \geqq 0$

holds.

$$
0<d<\frac{N(r, 1)}{N(r, 0)+N(r, f)} \text { for all } r \geqq r_{0}
$$

(4) There exist meromorphic functions $g, g \neq f$, with simple zeros $a_{v}^{\prime}$, simple ones $b_{v}^{\prime}$, simple poles $c_{v}^{\prime}(v=1,2,3, \ldots)$, and

$$
\begin{aligned}
& \left|a_{v}-a_{v}^{\prime}\right|=O\left(\exp \left(-\left|a_{v}\right|^{2 \varrho+\tau}\right)\right), \\
& \left|b_{v}-b_{v}^{\prime}\right|=O\left(\exp \left(-\left|b_{v}\right|^{2 \varrho+\tau}\right)\right), \\
& \left|c_{v}-c_{v}^{\prime}\right|=O\left(\exp \left(-\left|c_{v}\right|^{2 \varrho+\tau}\right)\right) .
\end{aligned}
$$

In view of the result on entire functions proved in [16] we will give the following remark: Theorem 2 does not hold for entire functions of order $\varrho<\infty$.

3. Some lemmas. To prove Theorem 1 and Theorem 2 we need two lemmas which are already published in [16] and [15]. We state them here without proofs.

Lemma 1 gives some information about the values taken by $f$ in small discs if the spherical derivative is growing very strongly. 
Lemma 1. Let be $f(z)$ a meromorphic function in $C$ of order $\varrho<\infty$. Let $z_{1}, z_{2}, \ldots$ be a sequence of points tending to infinity such that with some $\varepsilon>0$

$$
\lim _{v \rightarrow \infty} \exp \left(-\left|z_{v}\right|^{2_{\ell}+3 \varepsilon+1}\right) \frac{\left|f^{\prime}\left(z_{v}\right)\right|}{1+\left|f\left(z_{v}\right)\right|^{2}}=\infty
$$

holds. Then $f$ takes all values of the extended plane in each infinite subsequence of the discs $C_{v}=\left\{z|| z-z_{v} \mid<\exp \left(-\left|z_{v}\right|^{\varrho+\varepsilon}\right)\right\}$.

Lemma 2 gives some information about the size of the spherical derivative.

Lemma 2. Let $f$ be meromorphic in $\boldsymbol{C}$. Let two sequences of points $a_{1}, a_{2}, \ldots$ and $b_{1}, b_{2}, \ldots$ be given such that with two constants $K_{1}$ and $K_{2}$ one has

$$
\left|f\left(a_{v}\right)\right|<K_{1} \text { and }\left|f\left(a_{v}\right)-f\left(b_{v}\right)\right| \geqq K_{2} .
$$

Then on each straight line joining $a_{v}$ and $b_{v}$ there exists some point $z_{v}$ such that

$$
\left|a_{v}-b_{v}\right| \frac{\left|f^{\prime}\left(z_{v}\right)\right|}{1+\left|f\left(z_{v}\right)\right|^{2}} \geqq \frac{K_{2}}{1+\left(K_{1}+2 K_{2}\right)^{2}} \quad \text { for all } \quad v=1,2,3, \ldots .
$$

4. Proof of Theorem 1. To prove Theorem 1 we assume that there exists a meromorphic function $g$ with the zeros $a_{v}^{\prime}$, the ones $b_{v}^{\prime}$, and the poles $c_{v}^{\prime}(v=$ $1,2,3, \ldots)$, where all zeros, ones, and poles are simple. The order of $g$ is obviously equal to $\varrho$.

We will prove Theorem 1 by means of a contradiction to the assumed existence of $g$.

In the first part of the following proof we will assume additionally to (2.3) that

$$
0<\left|a_{v}-a_{v}^{\prime}\right|, \quad 0<\left|b_{v}-b_{v}^{\prime}\right| \text { and } 0<\left|c_{v}-c_{v}^{\prime}\right|
$$

hold. Later on we will remove these additional assumptions stepwise. We make here these additional assumptions to avoid the consideration of different cases. Define $\varepsilon>0$ by $\tau=1+4 \varepsilon$ and for $v=1,2,3, \ldots$

$$
\begin{aligned}
& C_{a_{v}}^{1}=\left\{z|| z-a_{v} \mid<2 \exp \left(-\left|a_{v}\right|^{2_{\ell}+1+4 \varepsilon}\right)\right\}, \\
& C_{b_{v}}^{1}=\left\{z|| z-b_{v} \mid<2 \exp \left(-\left|b_{v}\right|^{2 \varrho+1+4 \varepsilon}\right)\right\}, \\
& C_{c_{v}}^{1}=\left\{z|| z-c_{v} \mid<2 \exp \left(-\left|c_{v}\right|^{2 \varrho+1+4 \varepsilon}\right)\right\} .
\end{aligned}
$$

First we now claim that

$$
\begin{gathered}
0=\lim _{v \rightarrow \infty} \operatorname{Sup}\left\{|f(z)-1| \mid z \in C_{b_{v}}^{1}\right\}=\lim _{v \rightarrow \infty} \operatorname{Sup}\left\{|f(z)| \mid z \in C_{a_{v}}^{1}\right\} \\
=\lim _{v \rightarrow \infty} \operatorname{Sup}\left\{\frac{1}{|f(z)|} \mid z \in C_{c_{v}}^{1}\right\}
\end{gathered}
$$


and

$$
\begin{gathered}
0=\lim _{v \rightarrow \infty} \operatorname{Sup}\left\{|g(z)-1| \mid z \in C_{b_{v}}^{1}\right\}=\lim _{v \rightarrow \infty} \operatorname{Sup}\left\{|g(z)| \mid z \in C_{a_{v}}^{1}\right\} \\
=\lim _{v \rightarrow \infty} \operatorname{Sup}\left\{\left|\frac{1}{g(z)}\right| \mid z \in C_{c_{v}}^{1}\right\} .
\end{gathered}
$$

Assume that (4.2) does not hold. Without any restriction of generality we suppose in this case $0 \neq \lim _{v \rightarrow \infty} \operatorname{Sup}\left\{|f(z)| \mid z \in C_{a_{v}}^{1}\right\}$. Because there is one zero of $f$ in each of the discs $C_{a_{v}}^{1}$, Lemma 2 thus gives a sequence $z_{1}, z_{2}, z_{3}, \ldots \rightarrow \infty$ with $z_{\mu} \in C_{a_{v_{\mu}}}^{1}$ and

$$
2 \exp \left(-\left|a_{v_{\mu}}\right|^{2_{\varrho}+1+4 \varepsilon}\right) \frac{\left|f^{\prime}\left(z_{\mu}\right)\right|}{1+\mid f\left(z_{\mu}\right)^{2}} \geqq \delta>0
$$

with some fixed $\delta$. Hence it follows

$$
\lim _{\mu \rightarrow \infty} \exp \left(-\left|a_{v_{\mu}}\right|^{2 \varrho+1+3 \varepsilon}\right) \frac{\left|f^{\prime}\left(z_{\mu}\right)\right|}{1+\left|f\left(z_{\mu}\right)\right|^{2}}=\infty .
$$

Now by Lemma $1 f$ takes all values of the extended complex plane in the discs $\left\{z|| z-z_{\mu} \mid<\exp \left(-\left|a_{v_{\mu}}\right|^{\varrho+\varepsilon}\right)\right\}$. But this is contradictory to the supposition (2.2). Therefore (4.2) is true. Because of (2.3) the same argument gives (4.3).

From (4.2) and (4.3) it follows

$$
\lim _{v \rightarrow \infty} \operatorname{Sup}\left\{\left|\frac{g}{f}-1\right| \mid z \in C_{b_{v}}^{1}\right\}=\lim _{v \rightarrow \infty} \operatorname{Sup}\left\{\left|\frac{g-1}{f-1}-1\right| \mid z \in C_{a_{v}}^{1} \cup C_{c_{v}}^{1}\right\}=0 .
$$

Because of $f\left(a_{v}\right)=g\left(a_{v}^{\prime}\right)=0, f\left(b_{v}\right)=g\left(b_{v}^{\prime}\right)=1$ and $1 / f\left(c_{v}\right)=1 / g\left(c_{v}^{\prime}\right)=0$, one now sees that the function

$$
h=\frac{g}{f}-\frac{g-1}{f-1}
$$

takes the values $w_{1}$ and $w_{2}$ with $\left|w_{1}\right| \leqq 2$ and $\left|w_{2}\right| \geqq 3$ in all the discs $C_{a_{v}}^{1}, C_{b_{v}}^{1}$ and $C_{c_{v}}^{1}$ for all $v=1,2,3, \ldots$ If we now follow for $h$ and $g / f$ the arguments used to prove (4.2) we see that $h$ takes all values of the extended complex plane for almost all $v$ in each of the discs $C_{b}$ and $g / f$ in the discs $C_{a}$ and $C_{b}$ with

$$
\begin{aligned}
& C_{a_{v}}=\left\{z|| z-a_{v} \mid<\exp \left(-\left|a_{v}\right|^{\varrho+\varepsilon}\right)\right\}, \\
& C_{b_{v}}=\left\{z|| z-b_{v} \mid<\exp \left(-\left|b_{v}\right|^{\varrho+\varepsilon}\right)\right\}, \\
& C_{c_{v}}=\left\{z|| z-c_{v} \mid<\exp \left(-\left|c_{v}\right|^{\varrho+\varepsilon}\right)\right\} .
\end{aligned}
$$

Therefore in almost all of these discs $C_{b}$ there exists one zero of

$$
h(z)=\frac{g}{f}-\frac{g-1}{f-1}=\frac{f-g}{f(f-1)}=\frac{1-g / f}{f-1} .
$$

From the preceding follows, because of (2.2), that there exists at least one one-point of $g / f$ in almost all of the discs $C_{a_{v}}, C_{b_{v}}$ and $C_{c_{v}}$. For the rest of the proof of Theorem 1 
we use only this fact. But this is also true if we give up the additional supposition $0<\left|b_{v}-b_{v}^{\prime}\right|$ in (4.1). So we can replace (4.1) by

$$
0<\left|a_{v}-a_{v}^{\prime}\right| \text { and } 0<\left|c_{v}-c_{v}^{\prime}\right|
$$

and have

(4.5) almost all discs $C_{a_{v}}, C_{b_{v}}$ and $C_{c_{v}}$ contain at least one one-point of $g / f$.

In connection with (4.5) we point out that because of (2.2)

$$
\left\{\begin{array}{l}
C_{a_{\nu}} \cap\left(\left(\bigcup_{\substack{\mu=1 \\
\mu \neq \nu}}^{\infty} C_{a_{\mu}}\right) \cup\left(\bigcup_{\mu=1}^{\infty} C_{b_{\mu}}\right) \cup\left(\bigcup_{\mu=1}^{\infty} C_{c_{\mu}}\right)\right)=\emptyset, \\
C_{b_{v}} \cap\left(\left(\bigcup_{\mu=1}^{\infty} C_{a_{\mu}}\right) \cup\left(\bigcup_{\substack{\mu=1 \\
\mu \neq \nu}}^{\infty} C_{b_{\mu}}\right) \cup\left(\bigcup_{\mu=1}^{\infty} C_{c_{\mu}}\right)\right)=\emptyset, \\
C_{c_{v}} \cap\left(\left(\bigcup_{\mu=1}^{\infty} C_{a_{\mu}}\right) \cup\left(\bigcup_{\mu=1}^{\infty} C_{b}\right) \cup\left(\bigcup_{\substack{\mu=1 \\
\mu \neq \nu}}^{\infty} C_{c_{\mu}}\right)\right)=\emptyset
\end{array}\right.
$$

holds for all $v$.

We will use (4.5) and (4.6) later on.

Next we will look at $g / f$. For all $z \neq a_{v}, c_{v}^{\prime}$

and

$$
\left|\frac{z-a_{v}^{\prime}}{z-a_{v}}\right|=\left|\frac{\left(z-a_{v}\right)+\left(a_{v}-a_{v}^{\prime}\right)}{z-a_{v}}\right| \leqq 1+\frac{\left|a_{v}-a_{v}^{\prime}\right|}{\left|z-a_{v}\right|}
$$

$$
\left|\frac{z-c_{v}}{z-c_{v}^{\prime}}\right|=\left|\frac{\left(z-c_{v}^{\prime}\right)+\left(c_{v}^{\prime}-c_{v}\right)}{z-c_{v}^{\prime}}\right| \leqq 1+\frac{\left|c_{v}-c_{v}^{\prime}\right|}{\left|z-a_{v}\right|}
$$

hold. From this it follows that, if (2.3) holds, one has for almost all $v$ and for $z \notin$ $C_{a_{v}} \cup C_{c_{v}}$ with two constants $K_{1}$ and $K$ the relations

and

$$
\left|\frac{z-a_{v}^{\prime}}{z-a_{v}}\right| \leqq 1+K_{1} e^{-\left|a_{v}\right|^{2 e+\varepsilon}} e^{\left|a_{v}\right| e+\varepsilon} \leqq 1+K e^{-\left|a_{v}\right|^{2 e+\varepsilon}}
$$

$$
\left|\frac{z-c_{v}}{z-c_{v}^{\prime}}\right| \leqq 1+K_{1} e^{-\left|c_{v}\right|^{2 e+\varepsilon}} e^{\left|c_{v}\right|^{\mid e+\varepsilon}} \leqq 1+K e^{-\left|c_{v}\right|^{2 e+\varepsilon}} .
$$

Because the order of $f$ is equal to $\varrho$ and since $\varepsilon>0$, the product

$$
\prod_{v=1}^{\infty} \frac{z-a_{v}^{\prime}}{z-a_{v}} \prod_{v=1}^{\infty} \frac{z-c_{v}}{z-c_{v}^{\prime}}
$$

converges in $z \notin C^{*}=\bigcup_{v=1}^{\infty}\left(C_{a_{v}} \cup C_{c_{v}}\right)$ uniformly to a meromorphic function $h_{1}$ with order $\varrho_{1} \leqq \varrho$ and

$$
\lim _{\substack{|z| \rightarrow \infty \\ z \notin C^{*}}} h_{1}(z)=1 .
$$


Now

$$
h_{1} \frac{f}{g}
$$

is a meromorphic function of order $\varrho_{2} \leqq \varrho$ without zeros and poles. Therefore with some polynomial $p$ of degree $k \leqq \varrho$ we have

$$
\frac{g}{f}=h_{1} e^{p}
$$

By this and (4.6), for some sequence of reals $r$ tending to $\infty$ and some function $c(r)$ tending to 1 with $r \rightarrow \infty$ because of $\varrho \neq 1,2,3, \ldots$ and (2.3)

$$
\begin{aligned}
T\left(r, \frac{g}{f}\right) & \leqq c(r) m\left(r, e^{p}\right)+N\left(r, \frac{1}{f}\right)+N(r, g) \\
& =c(r) m\left(r, e^{p}\right)+N\left(r, \frac{1}{f}\right)+N(r, f)+o(N(r, f)) \\
& =\left(N\left(r, \frac{1}{f}\right)+N(r, f)\right)\left(1+o\left(N\left(r, \frac{1}{f}\right)+N(r, f)\right)\right) .
\end{aligned}
$$

Because of Nevanlinna's first fundamental theorem it follows from (4.5) and (4.6) that

$$
T\left(r, \frac{g}{f}\right)=T\left(r, \frac{1}{g / f-1}\right)+O(1) \geqq N(r, f)+N\left(r, \frac{1}{f-1}\right)+N\left(r, \frac{1}{f}\right)
$$

holds. Hence, because of (2.1) one must have for some sequence of reals $r$ tending to infinity

$$
\geqq\left(N\left(r, \frac{1}{f}\right)+N(r, f)\right)\left(1+\frac{N\left(r, \frac{1}{f-1}\right)}{N\left(r, \frac{1}{f}\right)+N(r, f)}\right) \geqq\left(N\left(r, \frac{1}{f}\right)+N(r, f)\right)(1+d) .
$$

This includes a contradiction because of $d>0$. Hence Theorem 1 is proved with the additional supposition (4.4).

We now consider the case that (4.4) is not fulfilled. In that case we have to replace the functions $f, 1 / f, g / f$ by $f^{*}, 1 / f^{*}, g^{*} / f^{*}$, respectively in (4.7), (4.8) and (4.9), where these functions are constructed by removing from $f$ and $g$ all common zeros and poles. Obviously

$$
N(r, f)+N\left(r, \frac{1}{f}\right) \geqq N\left(r, f^{*}\right)+N\left(r, \frac{1}{f^{*}}\right)
$$


and therefore instead of (2.1)

$$
0<d \leqq d_{1} \leqq \frac{N\left(f, \frac{1}{f-1}\right)}{N(r, 0)+N(r, f)} .
$$

Hence the contradiction in (4.9) exists also in the case that (4.4) is not fulfilled. Consequently, Theorem 1 is proved in all parts.

5. Proof of Theorem 2. Let some real $\varrho<\infty$ be given. Now to prove Theorem 2 choose some real $\tau>1$ and some sequence of points $a_{1}, a_{2}, \ldots$ with monotonic increasing modules, with $\varrho$ as the exponent of convergence and with sufficiently big $\left|a_{1}\right|$ as well as

$$
\left|a_{v+1}-a_{v}\right| \geqq 8 \exp \left(-\left|a_{v}\right|^{\varrho+\frac{1}{4}(\tau-1)}\right) .
$$

Then take a second sequence of points $b_{1}, b_{2}, \ldots$ with

Because of (5.1)

$$
\left|a_{v}-b_{v}\right| \leqq \exp \left(-\left|a_{v}\right|^{4 \varrho+2 \tau}\right) \text {. }
$$

$$
\left|b_{v+1}-b_{v}\right| \geqq 4 \exp \left(-\left|b_{v}\right|^{\varrho+\frac{1}{4}(\tau-1)}\right)
$$

holds. Thus

$$
f(z)=2 \prod_{v=1}^{\infty} \frac{z-a_{v}}{z-b_{v}}
$$

is a meromorphic function with

where $C^{*}=\bigcup_{v=1}^{\infty} C_{v}$ and

$$
\lim _{\substack{|z| \rightarrow \infty \\ z \notin C^{*}}} f(z)=2
$$

$$
C_{v}=\left\{z|| z-a_{v} \mid<\exp \left(-\left|a_{v}\right|^{2 \varrho+\tau}\right)\right\} .
$$

This follows immediately if one applies the construction used in the proof of Theorem 1 substituting $h_{1}$ with $f$. Because of (5.3) and since $\left|a_{1}\right|$ was chosen sufficiently big, there is in each disc $C_{v}$ exactly one one-point $c_{v}$ from $f(z)$ while outside $C^{*}$ there is no solution of $f(z)=1$. Hence especially

$$
\left|c_{v+1}-c_{v}\right| \geqq 2 \exp \left(-\left|c_{v}\right|^{\varrho+\frac{1}{4}(\tau-1)}\right)
$$

holds. Thus $f$ fulfils the conditions (1) and (2) of Theorem 2. Again the construction of the zeros, ones and poles of $f$ gives obviously (3) of Theorem 2 because all these zeros, ones and poles are very close together in relation to their exponent of convergence. If one now chooses two sequences of points $a_{1}^{\prime}, a_{2}^{\prime}, \ldots$ and $b_{1}^{\prime}, b_{2}^{\prime}, \ldots$ with

$$
0 \neq\left|a_{v}-a_{v}^{\prime}\right|<\exp \left(-\left|a_{v}\right|^{-4 \varrho+2 \tau}\right)
$$


and

then the function

$$
0 \neq\left|b_{v}-b_{v}^{\prime}\right|<\exp \left(-\left|a_{v}\right|^{-4 \varrho+2 \tau}\right)
$$

$$
g(z)=2 \prod_{n=1}^{\infty} \frac{z-a_{v}^{\prime}}{z-b_{v}^{\prime}}
$$

will obviously satisfy the assertion (4) of Theorem 2. Hence Theorem 2 has been proved.

As it was announced in the introduction of this paper, we can now easily see that such examples as we just constructed to prove Theorem 2 do not exist in the case of entire functions: We are able to prove Theorem 2 by choosing the zeros and poles of the function so close together that the suppositions of Lemma 1 are fulfilled. From Lemma 1 we see that the function $f$ has to take all values of the extended complex plane and therefore especially the value one. But just this method cannot be applied to entire functions, because every entire function has naturally one exceptional value. In other words: there exists no entire function of prescribed order where zeros and ones are as close together as in the case of Theorem 2.

\section{References}

[1] Anderson, J. M., and J. Clunie: Picard sets of entire and meromorphic functions. - Ann. Acad. Sci. Fenn. Ser. A I Math. 5, 1980, 27-43.

[2] Baker, I. N.: Linear Picard sets for entire functions. - Math. Nachr. 64, 1974, 263-276.

[3] BAKer, I. N., and L. S. O. Liverpool: Further results on Picard sets of entire functions. Proc. London Math. Soc. 26, 1973, 82-98.

[4] Lehto, O.: A generalization of Picard's theorem. - Ark. Mat. 3, 1958, 495-500.

[5] NevanlinNa, R.: Über die Konstruktion von meromorphen Funktionen mit gegebenen Wertzuordnungen. - Festschrift zur Gedächtnisfeier für Karl Weierstraß, 1815-1965, Westdeutscher Verlag, Köln—Opladen, 1966, 579—582.

[6] Матsumoto, K.: Remark to Lehto's paper “A generalization of Picard's theorem”. - Proc. Japan Acad. 38, 1962, 636-640.

[7] Ozawa, M.: On the zero-one set of an entire function. - Kōdai Math. Sem. Rep. 28, 1977, $311-316$.

[8] Ozawa, M., and K. NiIno: Deficiencies of an entire algebroid function. - Kōdai Math. Sem. Rep. 22, 1970, 98-113.

[9] Rubel, L. A., and C. C. YANG.: Interpolation and unavoidable families of meromorphic functions. - Michigan Math. J. 20, 1973, 289-296.

[10] Toppila, S.: Picard sets for meromorphic functions. - Ann. Acad. Sci. Fenn. Ser. A I Math. $417,1967,1-24$.

[11] Toppila, S.: Some remarks on the value distribution of entire functions. - Ann. Acad. Sci. Fenn. Ser. A I Math. 421, 1968, 1-11.

[12] ToppILA, S.: On the value distribution of integral functions. - Ann. Acad. Sci. Fenn. Ser. A I Math. 574, 1974, 1-20.

[13] Toppila, S.: Linear Picard sets for entire functions. - Ann. Acad. Sci. Fenn. Ser. A I Math. 1, 1975, 111-123. 
[14] WinkLer, J.: Über Picardmengen ganzer und meromorpher Funktionen. - Math. Z. 109, 1969, 191-204.

[15] WiNkLeR, J.: Über Picardmengen ganzer Funktionen. - Manuscripta Math. 1, 1969, 191-199.

[16] WiNKLER, J.: Zur Existenz ganzer Funktionen bei vorgegebener Menge der Nullstellen und Einsstellen. - Math. Z. 168, 1979, 77-85.

Technische Universität Berlin

Fachbereich Mathematik

D 1000 Berlin 12

Federal Republic of Germany

Received 8 November 1983 